\title{
Le syndrome de Gorlin-Goltz : rapport d'un cas
}

\section{Gorlin Goltz syndrome: report of a case}

\section{MOTS-CLEFS :}

- Keratokyste odontogénique,

Syndrome de

Gorlin-Goltz,

Kystes des Maxillaires

\section{KEYWORDS:}

- Keratocystic odontogenic, Gorlin-Goltz Syndrome, Maxillary cysts

AOS 2014;270:18-22 DOI: $10.1051 / \mathrm{aos} / 2014404$ (C) EDP Sciences 2014

\section{Résumé}

Les auteurs présentent la prise en charge d'un patient atteint d'une polykystose maxillomandibulaire. Compte tenu des antécédents familiaux et du diagnostic histologique qui met en évidence des kératokystes multiples, on évoque chez ce patient une forme du syndrome de Gorlin-Goltz.

\section{Abstract}

The authors present one patient with multiple oral cysts. The formily history and the histological diagnostic showing multiple keratocysts, we suspect one form of Gorlin-Goltz syndrome.

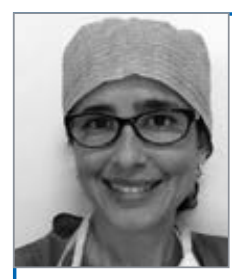

- Monica Umana, Chirurgien-Dentiste

DU Chirurgie et Prothèses Implantologiques Paris 5

DIU Pathologie de la Muqueuse Buccal Paris 6-Montpellier I

DU Chirurgie Buccal Montpellier I

Thierry Piral, Chirurgien maxillo-facial, Stomatologiste, Paris 


\section{INTRODUCTION}

La nævomatose baso-cellulaire (NBC), aussi connue sous le nom de syndrome de Gorlin-Goltz, est une affection héréditaire rare appartenant à la famille des neurocristopathies ou maladies par anomalies des crêtes neurales [1]. Son mode de transmission est autosomique dominante à pénétrance complète et à expressivité variable. Bien que ce syndrome ait été décrit pour la première fois en 1894 par Jarisch et White $[2,3]$, ce n'est qu'en 1960 que Gorlin et Goltz [4] ont déterminé la triade qui établit le diagnostic : multiples carcinomes basocellulaires, kératokystes odontogéniques des maxillaires et anomalies costales. À cette triade peuvent s'ajouter d'autres anomalies très variées : osseuses, cutanées, neurologiques, oculaires, endocriniennes et génitales [5] qui apparaissent en général de façon décalée dans le temps. L'incidence de ce syndrome est estimée entre 1/50 000 à 1/150 000 dans la population générale. Les deux sexes sont également atteints [6]. C'est au sein de la race caucasienne que la majorité des cas a été rapportée [7].
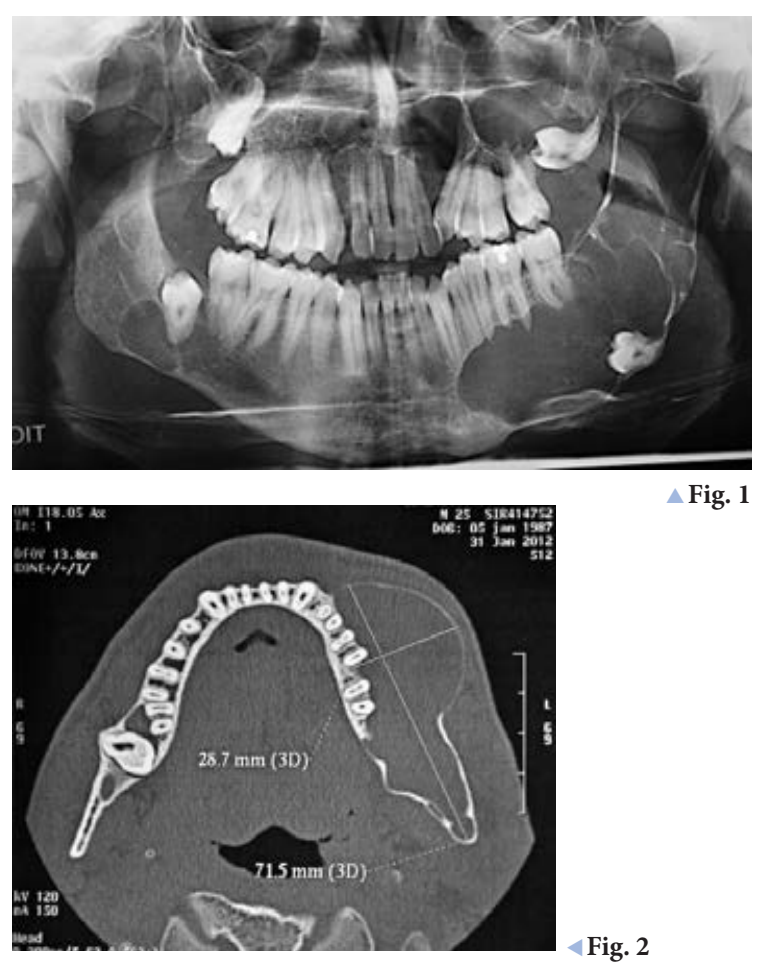

$\triangle$ Fig. 1

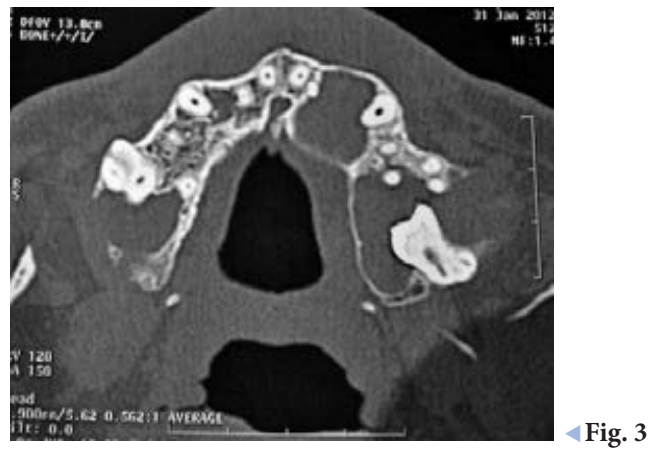

\section{OBSERVATION}

Cet article rapporte le cas d'un homme de 26 ans, adressé par son chirurgien-dentiste traitant pour la prise en charge d'une tuméfaction vestibulaire, apparue en l'espace de quelques semaines en regard de la région prémolaire et molaire mandibulaire gauche.

Lanamnèse ne révèle pas d'antécédents médicaux particuliers.

Antécédents familiaux : aucune manifestation clinique du syndrome n’a été confirmée chez les parents. Dans la fratrie au nombre de six ( 2 frères et 4 sœurs), deux sœurs présentent des manifestations similaires avec présence de multiples kératokystes maxillaires.

L'examen extra-oral montre une déformation de la joue gauche. La sensibilité de la région labio-mentonnière est normale.

L'examen endo-buccal révèle l'existence d'une tuméfaction vestibulaire recouverte d'une muqueuse d'aspect normal, sétendant de la partie distale de la dent 33 jusquà la région de la dent 38 et comblant le fond du vestibule.

À la palpation, la tuméfaction était indolore, de consistance ferme mais dépressible. Les dents en rapport étaient saines et répondaient positivement au test de vitalité. Aucune mobilité dentaire nétait constatée.

La palpation cervicale ne retrouve aucune adénopathie. L'examen radiographique par orthopantomogramme (fig. 1) dévoile l'existence de multiples zones radioclaires d'aspect ostéolytique. Au maxillaire, elles apparaissent centrées sur la dent 18 , entre les dents 12 et 13 , 22 et 23 , ainsi que sur la dent 28 . À la mandibule, la radiographie montre à gauche une lésion en regard de la région des prémolaires et molaires et sur la dent 48 également.

L'examen tomodensitométrique confirme la présence d'une importante lésion ostéolytique de 71,5 mm de longueur sur $28,7 \mathrm{~mm}$ dépaisseur située entre les dents 33 et 38 . Les coupes coronales du scanner montrent également que les corticales vestibulaire et linguale sont soufflées.

Étant donné ces renseignements cliniques, l'intervention se fait sous anesthésie générale et permet l'exérèse des foyers lésionnels avec l'avulsion des dents 18, 28, 38 et 48 présentant un rapport direct avec les lésions ostéolytiques (fig. 2 et 3).Lexamen anatomopathologique a retrouvé des formations kystiques bordées par un épithélium malpighien kératinisé régulier. La kératinisation était de manière prédominante parakératosique. L'examen conclut à la présence de kératokystes multiples.

Examens complémentaires :

la radiographie du thorax ne montre aucune anomalie. Les images cliniques et radiographiques de contrôle 
réalisées six mois plus tard montrent une cicatrisation osseuse et muqueuse (fig. 4).

Aucune récidive ne s'est produite. Le patient reste néanmoins toujours sous surveillance. Un scanner de contrôle du maxillaire inférieur, effectué un an
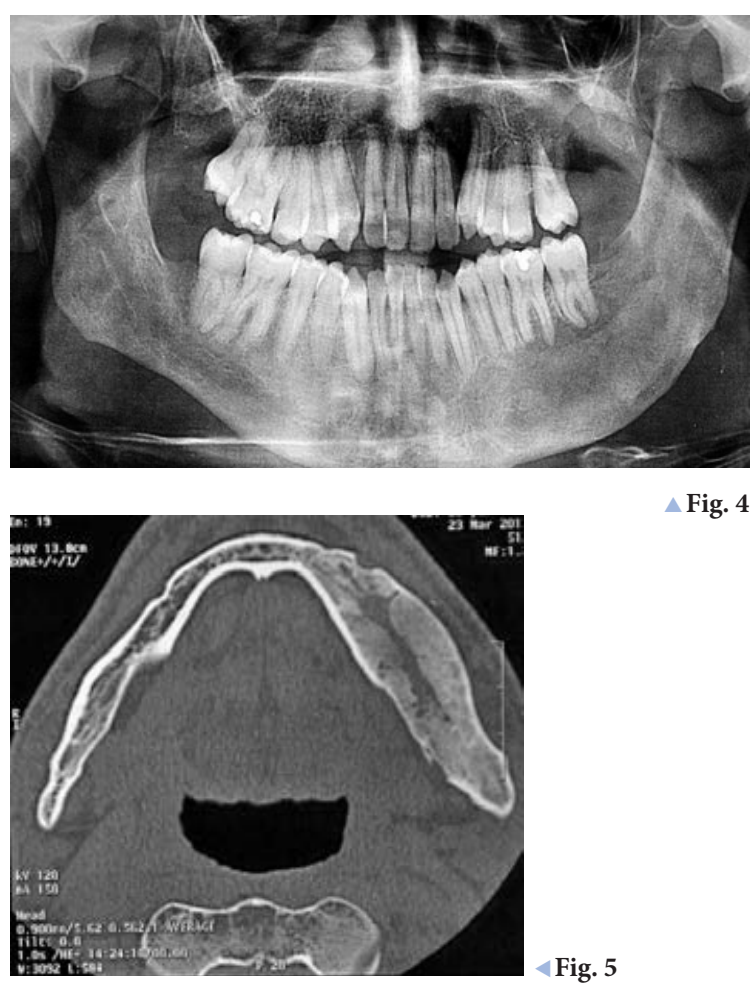

$\triangle$ Fig. 4

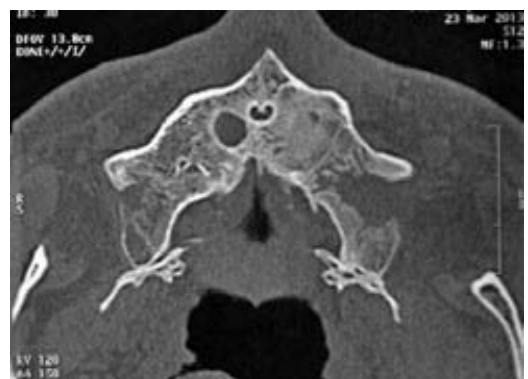

Fig. 6 après l'intervention chirurgicale, met en évidence le remodelage osseux et une légère asymétrie de l'arc mandibulaire (fig. 5). Au niveau maxillaire supérieur, on note également la présence d'os en néoformation. Cependant un doute existe sur la persistance d'un kyste maxillaire droit de $7 \mathrm{~mm}$ de diamètre sur $6 \mathrm{~mm}$ de hauteur (fig. 6). Un scanner est prévu dans 1 an, il permettra de voir si cette lésion est évolutive ou s'il s'agit d'un stigmate chirurgical.

\section{DISCUSSION}

Le syndrome de Gorlin-Goltz est une maladie héréditaire à transmission autosomique dominante qui fait partie des neurocristopathies, il s'agit de pathologies génétiques touchant avec prédilection des éléments issus de la crête neurale. Le tableau clinique complet touche prioritairement la peau, les yeux, les os, le système nerveux et le système endocrinien [1].

La maladie se manifeste donc par de très nombreux symptômes, variables d'un patient à l'autre. Il existe plus de quarante critères de diagnostic. Néanmoins, chaque patient atteint de cette maladie ne présente pas l'ensemble des critères [8]. Les critères de diagnostic pour NBC ont été établis par Evans et al. [9], et modifiés par Kimonis et al. en 1973 [10]. Ils ont été classés en deux catégories, majeure et mineure, en fonction de leur fréquence d'apparition. Il est possible de poser un diagnostic de nævomatose baso-cellulaire lorsqu'un patient présente au minimum deux critères majeurs ou bien associe un critère majeur à deux critères mineurs [11] (tableau I).

Dans le cas présenté, le diagnostic de syndrome de Gorlin-Goltz est hautement suspecté puisque deux critères majeurs sont présents : kératokystes multiples

₹ Tableau 1 : Critères du diagnostic du syndrome de Gorlin $[12,13]$

Critères majeurs
1. Plus de 2 carcinomes baso-cellulaires ou un carcinome baso-cellulaire avant 20 ans
3. Au moins 3 ponctuations palmoplantaires
4. Calcifications bilamellaires de la faux du cerveau
5. Côtes bifides, fusionnées ou écartés
6. Parent au $1^{\text {er }}$ degré atteint du syndrome
Critères mineurs
1. Macrocéphalie
2. Malformation congénitale : fente labiale ou palatine, bosses frontales, hypertélorisme, traits grossiers
3. Autres anomalies du squelette: déformation de Sprengel des omoplates, déformation pectorale, syndactylie
4. Anomalies RX : selle turcique pontée, hémivertèbres, fusion de corps vertébraux, anomalies des mains
et des pieds, anomalies des phalanges (liseré en « flamme »)
5. Fibromes ovariens
6. Médulloblastome (surtout si desmoplasique)


et antécédents familiaux (3 enfants atteints sur une fratrie de six). Les parents n’ont pas pu être examinés. Le kératokyste odontogénique (KO) représente entre 10 et $20 \%$ de l'ensemble des lésions kystiques des maxillaires. Cette fréquence le place en troisième position des kystes odontogéniques derrière le kyste radiculaire et le kyste dentigère selon la classification de l'OMS [14].

Lorsqu'il se présente de façon isolée - sans association avec le syndrome -, il survient principalement au cours des deuxièmes et troisièmes décennies $[15,16]$, ce qui est le cas chez le patient présenté. En revanche, chez les patients atteints du syndrome de Gorlin-Goltz, les kératokystes apparaissent plus tôt, pendant la première décennie, ils représentent souvent la première manifestation de la maladie [17]. Généralement, la découverte est fortuite, à loccasion d'un examen radiographique de routine.

Les KO isolés sont localisés plus fréquemment (65-83\%) dans la mandibule, notamment au niveau de la région de la troisième molaire, de l'angle ou de la branche montante [18].Au maxillaire, ils apparaissent spécialement dans les régions des dents de sagesse et incisivo-canines [19]. Lorsqu'ils sont associés au syndrome, les kératokystes peuvent être retrouvés dans n’importe quelle localisation des maxillaires.

À l'examen radiologique, on peut observer de multiples lésions rondes ou ovales exhibant une image uni- ou multiloculaire, au contour régulier avec des bords sclérotiques. Elles peuvent être uni- ou bilatérales [17]. Le kératokyste peut quelquefois prendre la forme d'une vaste géode unique festonnée, déformant les corticales amincies (surtout la corticale externe), envahissant chaque segment osseux mais respectant le condyle [20,21].Sur le plan histologique, le KO se caractérise par une couche épithéliale uniforme sans corps muqueux de Malpighi, une lumière parakératinisée ou orthokératinisée ondulée et une importante couche de cellules basales [22, 23].

La présentation clinique des KO est variable. Initialement, ils sont asymptomatiques. Ils deviennent symptomatiques en cas de surinfection ou de croissance importante qui provoquent une déformation osseuse et le déplacement des dents adjacentes [24]. Le kyste souvent volumineux contient un liquide crémeux et englobe une dent incluse.

D’autre part, le KO reste une tumeur bénigne malgré son potentiel agressif local avec envahissement des structures voisines, en particulier osseuses. On peut également observer le développement d'un carcinome épidermoïde sur la paroi kystique [14].

Sur le plan thérapeutique, la chirurgie est le seul traitement envisageable : elle doit permettre lénucléation totale de la lésion.

Étant donné le taux élevé des récidives parfois assez tardives - jusquà $60 \%$ selon les séries - il est impératif de réaliser des suivis cliniques et radiologiques annuels au-delà des cinq premières années après l'ablation chirurgicale de la tumeur, éventuellement même à vie [25].

Les principaux diagnostics différentiels sont représentés par le kyste dentigère, le kyste radiculaire et l'améloblastome. Ces lésions peuvent prendre un aspect radiologique similaire et seul l'examen histologique permet de poser le diagnostic [14].

\section{CONCLUSION}

La nævomatose baso-cellulaire (NBC) ou syndrome de Gorlin est une affection héréditaire rare mais pas exceptionnelle qui doit être connue. Lors de la réalisation d'une radiographie panoramique de routine, des kératokystes odontogéniques peuvent être détectés ; ils sont souvent une des premières manifestations cliniques de la maladie. L'examen clinique et les examens radiographiques doivent être complétés par l'anamnèse familiale qui présente une importance clé. Dans le cas présenté, le diagnostic de syndrome de Gorlin-Goltz est hautement suspecté puisque deux critères majeurs sont présents : kératokystes multiples et antécédents familiaux ( 3 enfants atteints sur une fratrie de six). Les parents n'ont pas pu être examinés. Compte tenu du polymorphisme du syndrome, sa prise en charge doit être effectuée par une équipe interdisciplinaire [25].
Bibliographie

[1] Quatresooz P, Vandenbossche G., PiérardFranchimont C., Piérard G. La peau et ses principales neurocristopathies. Rev Med Liege 2008; 63 (5-6): 354-358.
[2] Jarisch W. Zur lehre von den hautgeschwulsten. Archiv für Dermatologie und Syphilis 1894; (28):163-165.

[3] White JC. Multiple benign cystic ephiteliomata. J Cutan Dis. 1894; (12):477-481.

[4] Gorlin RJ, Goltz RW: Multiple nevoid basal cell epithelioma, jaw cysts and bifid rib: A syndrome. New Engl J Med 1960; (262):908-912.
[5] Casaroto AR, Rocha Loures DCN, Moreschi E. Early diagnosis of Gorlin-Goltz syndrome: case report Head and Face Medicine 2011; 7 (2).

[6] Patil K, Mahima VG, and Gupta B. Gorlin syndrome: a case report. Journal of Indian Society of Pedodontics and Preventive Dentistry, 2005; 23 (4):198-203. 


\section{Bibliographie}

[7] Maudit G, Plauchu H. La nævomatose basocellulaire. Étude génétique et fréquence des cas sporadiques. Lyon Médical, 1982 ; 247 (7) 353-358.

[8] D’Agostino C. Naevomatose Basocellulaire ou Syndrome de Gorlin-Goltz : Rôle de lodontologiste. Th: Chir- Dent. ; Université Henri Poincaré, Nancy I 2010 : 11.

[9] Evans DGR, Ladusans EJ, Rimmer S, Burnell LD, Thakker N et Farndon PA. Complications of the naevoid basal cell carcinoma syndrome: results of a population based study. Journal of Medical Genetics 1993; 30 (6): 460-464

[10] Kimonis VE, Goldstein AM, Pastakia B. Clinical manifestations in 105 persons With nevoid basal cell carcinoma syndrome. American Journal of Medical Genetics 1997; (69) 299-308.

[11] Laborde-Pedel C. Le syndrôme de Gorlin. Th : Chir-Dent.; Université de Reims 1994 ; 114.

[12] Abadie C, Gauthier-Villars M, Sirvent N, Coupier I. Oncogénétique en oncopédiatrie. Arch Pédiatr 2012; (19) :863-875.
[13] Gosavi K, Mundada SD. Anaesthetic management in Gorlin-Goltz syndrome. Indian J Anaesth 2012; (56): 394-396.

[14] Favre-Dauvergne E, Auriol M, Le Charpentier Y. Kystes des maxillaires. Encycl Méd Chir ,Paris : Stomatologie-Odontologie I 1994;22-062-G-10:6.

[15] Manfredi M, Vescovi P, Bonanini M, Porter S. Nevoid basal cell carcinoma syndrome: a review of the literature. Int J Oral Maxillofac Surg 2004; 33 (2):117-124.

[16] Reyes Macias JF, Bagán Sebastián JV. Síndrome de Gorlin-Goltz : Revisión de la literatura y reporte de un caso. Rev Europ Odonto Estomatol 2002; 14(2):105-112.

[17] Ortega-García-de Amezaga A, GarcíaArregui O, Zepeda-Nuño S, Acha-Sagredo A, Aguirre-Urizar JM. Gorlin-Goltz syndrome: Clinicopathologic aspects. Med Oral Patol Oral Cir Bucal 2008; 13(6):E338-343

[18] Barnes L, Eveson JW, Reichart P, Sidransky D. Pathology and genetics of head and neck tumours. Lyon: IARC Press; 2005. WHO classification of tumours series.

[19] Ali M, Baughman RA. Maxillary odontogenic keratokyst. A common and serious clinical misdiagnosis. JADA 2003; (134):877-883.
[20] Kissi L, Benyahya I, Rifki A. Le kératokyste isolé : A propos d'un cas. Journal de l'Ordre des dentistes du Québec 2006;(43):321328.

[21] Sauveur G, Ferkdadji L, Gilbert E, Mesbah M. Kystes des maxillaires. Elsevier Masson, EMC Médecine buccale 2008 , 28-550-G-10.

[22] Myoung H, Hong SP, Hong SD, Lee JI, Lim CY, Choung PH, et al. Odontogenic keratocyst: review of 256 cases for recurrence and clinicopathologic parameters. Oral Surg Oral Med Oral Pathol Oral Radiol Endod 2001; 91(3):328-333

[23] Coleman H, Altini M, Ali H, Doglioni C, Favia G, Maiorano E. Use of calretinin in the differential diagnosis of unicystic ameloblastomas. Histopathology 2001; 38 (4):312-317.

[24] Goyault G, Moser T, Lutz JC, Neuville A, Buy X, Freitas R, Roy C, Gangi A. Keratokyste odontogénique. Journal de radiologie 2007;(88) 1733-1735.

[25] Bornstein MM, Filippi A, Altermatt HJ, Lambrecht JT, Buser D. Le kératokyste odontogène : kyste odontogène ou tumeur bénigne. Rev Mens Suisse Odontostomatol 2005; 115 (2):110-128.

\section{Chirurgie osseuse préimplantaire}

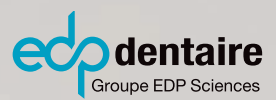

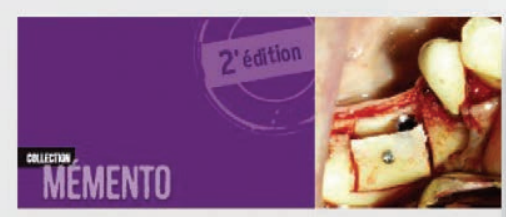

Chirurgie osseuse
préimplantaire

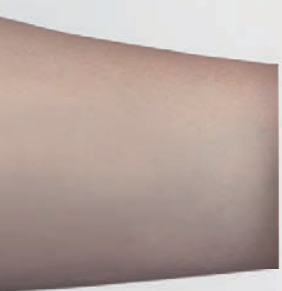

\section{Guy. Princ et al.}

Les techniques chirurgicales osseuses préimplantaires ont considérablement évolué ces dernières années. Ce " Mémento ", qui en est à sa deuxième édition, a pour objectif de les préciser de façon synthétique et claire. Il doit permettre à l'odontologiste et au chirurgien oral leur parfaite intégration pour mener à bien le plan du traitement. L'ensemble des chapitres passe en revue, de façon exhaustive, tous les points fondamentaux inhérents à ces techniques.

138 pages, 50 euros. 